\title{
Anomalous Linear Layer-dependent Blue Shift of Interband Transition in Two- Dimensional Materials
}

Xingxu Yan ${ }^{1}$, Feng Xue ${ }^{2}$, Chaitanya Gadre ${ }^{1}$, Zhe Wang ${ }^{2}$, Ruqian Wu ${ }^{1}$, Peng Wang ${ }^{3}$ and Xiaoqing Pan ${ }^{1}$

${ }^{1}$ University of California - Irvine, Irvine, California, United States, ${ }^{2}$ Fudan University, Shanghai, Shanghai, China (People's Republic), ${ }^{3}$ Nanjing University, Nanjing, Jiangsu, China (People's Republic)

Monochromated electron energy loss spectroscopies (EELS) offer direct interrogations of the local electronic structure for two-dimensional (2D) semiconducting materials, including emerging transitionmetal dichalcogenides (TMDCs) and their complex heterostructures [1,2]. Unique electronic structures of 2D TMDCs pave the way for abundant cutting-edge applications, including electronic devices and optoelectronic devices [3]. The bandgap excitations are nonlinearly red-shifted with the increasing layer number, leading to an intriguing tunable photon response in the visible-light range [4]. However, there is a lack of systematic study on the dielectric response of 2D TMDCs in the ultraviolet (UV) range. Typical optical approaches for measuring the electronic structure, such as absorption, photoluminescence, and photo-conductivity spectroscopies, reach the spatial resolution in the order of micrometer, which is insufficient to accurately identify nanoscale features in a complex 2D TMDC heterostructure. Another issue for detecting UV-range photoresponse is the recorded energy range being limited by the incident photon energy (typically $2.33 \mathrm{eV}$ ). By combining scanning transmission electron microscopy (STEM) with valence EELS (VEELS) method, we probed the local dielectric response of MoS2 nanostructures with a terrace-like structure from the visible-light to UV range [5].

Fig. 1 illustrates the spatial resolution of VEELS on a triangle-morphology 1L-2L heterojunction structure of AB-stacked $\mathrm{MoS}_{2}$. The energy resolution of monochromated EELS was about $0.1 \mathrm{eV}$. Averaged VEEL spectra in Fig. 1(b) show two major peaks of A,B excitation at about $2.1 \mathrm{eV}$ and interband transition peak around $3.1 \mathrm{eV}$. The major diff erence between $1 \mathrm{~L}$ and $2 \mathrm{~L}$ spectra is the energy shift of the interband transition peak from $3.17 \mathrm{eV}$ for monolayer one to $3.19 \mathrm{eV}$ for bilayer one. Figs. 1 (c) and (d) display 2D mappings of the spectral weights of $1 \mathrm{~L}$ and $2 \mathrm{~L}$ reference spectra respectively after applying the multiple least-squares method on the spectrum image data. The dielectric response can be well separated even with one layer difference by VEELS approach. A further line-scan VEELS data sets were acquired across two interfaces between 1L and 2L. The spectral weight signals in Fig. 1(f) are in agreement with the ADF signal, but have $1.6 \mathrm{~nm}$ broadening, compared to the ADF signal. This phenomenon can be explained by the quantum mechanical approach, which calculated the probability of dipolar excitation and provided a delocalization of 1-4 $\mathrm{nm}$ for an energy loss of $3 \mathrm{eV}$ [6]. Therefore, VEELS method can offer nanometer spatial resolution, far superior to those of traditional optical methods.

Then, a systematic investigation of the layer dependence was conducted by recording valence EEL spectra of various samples with different thickness in the energy range of 1-10 eV as shown in Fig. 2(a). Fig. 2(b) plots the peak centers of A,B excitaton and interband transition as a function of layer number. As the layer number increases, A,B excitonic peaks undergo a slight red shift due to bandgap shrinkage and reduction of the exciton binding energy. Interestingly, the interband transition peak at 3.0-3.5 eV shows an anomalous linear layer-dependent blue shift, indicating the non-negligible interlayer interaction in $\mathrm{MoS}_{2}$ flakes. The slope was fitted as $18.6 \pm 1.0 \mathrm{meV} /$ layer. First-principles calculations revealed that the blue shift of the interband transition was due to the change of the second band below the Fermi level around the $\Gamma$ point induced by the increased interlayer van der Waals interaction. Meanwhile, the electronic 
structure is insensitive to either AA or AB stacking order. The tunable interband transition has a potential application for modulating ultraviolet-light devices [7].
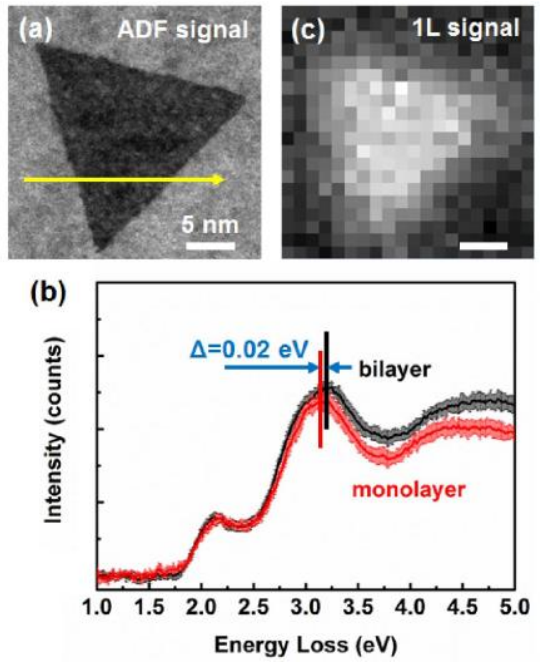
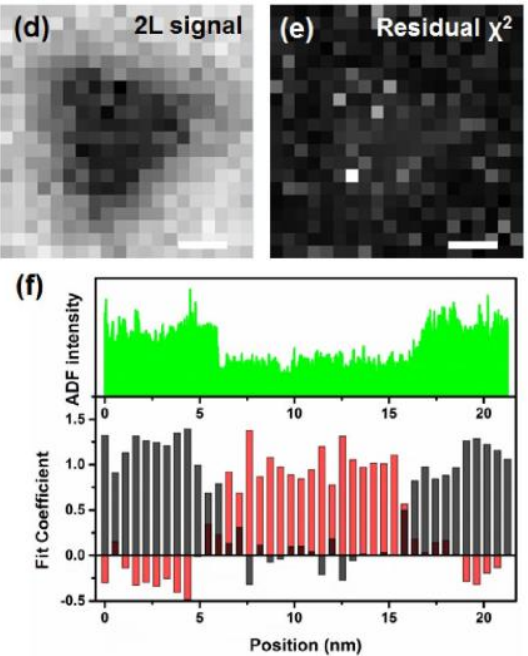

Figure 1. VEELS analysis of 1L-2L AB-stacked MoS2. (a) ADF-STEM image of triangle- morphology 1L-2L AB-stacked MoS2. (b) Average VEEL spectra of 1L (average of 14 spectra) and 2L areas (average of 11 spectra). (c, d) Mappings of fit coefficient values for 1L (c) and 2L VEELS (d) respectively, from the same area in (a), where the intensity is corresponding to the fit coefficient by the multiple least squares method. (e) Mapping of residual $\chi 2$ signals. (f) Comparison of ADF intensity (upper panel), line profile of fit coefficients (bottom panel) of 1L (red) and 2L (black) signals along the yellow arrow in (a).
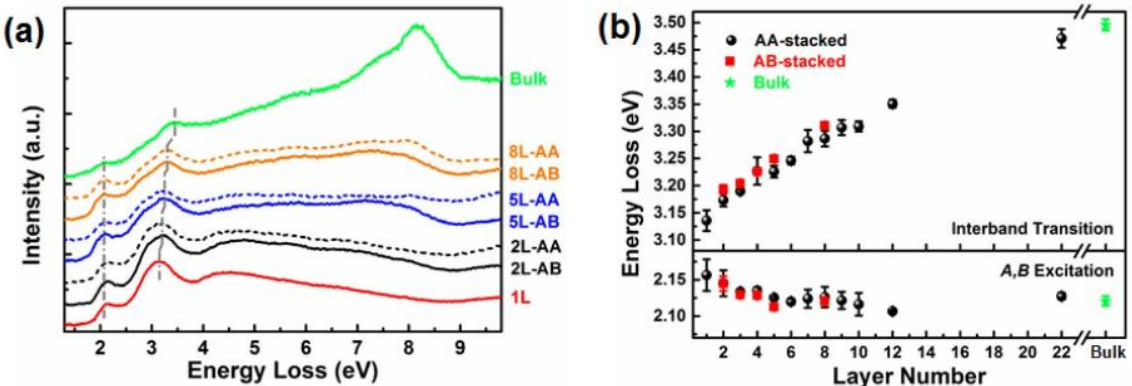

Figure 2. VEEL spectra and electronic structures from MoS2 with various layer numbers. (a) Representative VEEL spectra of 1L, 2L, 5L, 8L, and bulk MoS2 areas with AB (solid line) and AA (dashed line) stacking orders. The positions of the A,B excitation and interband transition are marked with gray dashed lines. (b) Layer dependence of the energy loss value of the interband transition and A,B excitation on the layer number.

\section{References}

[1] M. Y. Li et al., Science 349 (2015), p. 524-528.

[2] J. H. Hong et al., Phys. Rev. B 93 (2016), 075440.

[3] K. F. Mak et al., Nat. Nanotechnol. 7 (2012), p. 494-498.

[4] K. F. Mak et al., Phys. Rev. Lett. 105 (2010), 136805.

[5] X.X. Yan et al., J. Phys. Chem. C 124 (2020), p. 1609-1616.

[6] R. Erni and N. D. Browning, Ultramicroscopy 104 (2005), p. 176-192.

[7] This work was supported by the Department of Energy (DOE) under Grant DE-SC0014430. TEM experiments were conducted using the facilities in the Irvine Materials Research Institute (IMRI) at the University of California - Irvine. 